\title{
Variational Iteration Method for Solving Two Dimensional Volterra - Fredholm Nonlinear Integral Equations
}

\author{
M. H. Saleh \\ Mathematics Department, \\ Faculty of Science, \\ Zagazig University, Zagazig, \\ Egypt
}

\author{
D. Sh. Mohamed \\ Mathematics Department, \\ Faculty of Science, \\ Zagazig University, Zagazig, \\ Egypt
}

\author{
R. A. Taher \\ Mathematics Department, \\ Faculty of Science, \\ Zagazig University, Zagazig, \\ Egypt
}

\begin{abstract}
In this paper we investigate the numerical solution of two dimensional Volterra - Fredholm integralequations by Variational iteration method. Two numerical examples are given to illustrate themethod.
\end{abstract}

\section{Keywords}

Variational iteration method ,Volterra-fredholm ,Lagrange multiplier ,Two dimensional equations.

\section{INTRODUCTION}

Many works have been focusing on the development of more advanced and efficients methods for the two dimensional nonlinear Volterra integral equations such as Collocation method, Legendre polynomials, Tau method, Iterative method ,Extrapolation methods and others see [1 - 10] .

In 1987 Inokuti in [17 ] proposed a general Lagrange multiplier method for solving nonlinear operator

forms as follows :

$\mathrm{Lu}+\mathrm{NU}=\mathrm{g}(\mathrm{x})$

where $\mathrm{L}$ is a linear operator, $\mathrm{N}$ is a nonlinear operator, $\mathrm{g}(\mathrm{x})$ is a known analytic function and $\mathrm{u}$ is

an unknown function that to be determind. Also on the supposition that $\mathrm{u} 0$ is the solution of $\mathrm{LU}=0$,

It can be found via Variational theorem in $[12,17]$.The Inkuti method is modified by he which it can be written

$$
u_{n+1}\left(x_{0}\right)=u_{n}\left(x_{0}\right)+\int_{0}^{x_{0}} \lambda\left(L u_{n}+N u_{n}-g\right) d s
$$

where $\mathrm{u} 0$ is an initial approximatation and un is are stricted variation , in other words $\delta u n=0$ in[12] , for arbitrary of $x 0$ ,author has converted Eq.(2) to the following equation [13, 14].

$u_{n+1}(x)=u_{n}(x)+\int_{0}^{x} \lambda\left(L u_{n}(s)+N u_{n}(s)-g(s)\right) d s$

the above integeral in Eq.(3) is called a correction function and index $\mathrm{n}$ denotes the $\mathrm{nth}$ approximation. Also Eq.(3)is called Variational iteration method (VIM). In $[15,16]$ this method is used for solving nonlinear problems .The Variational iteration method is effective and easy for linear problem because

exact solution can be given by one iteration. In the above process Eq.(3) is written in following form

$$
u_{n+1}(x)=u_{n}(x)+\int_{0}^{x} \lambda\left(L u_{n}(s)+N u_{n}(s)-g(s)\right) d s
$$

In Eq.(4) by using $\mathrm{u} 0(\mathrm{x})$ as an initial approximation, we obtain a sequence of approximations of exact solution of Eq.(1). for illustration of effectively, easily and accurately a large class of nonlinear problems with approximations which converge quickly, Exact solution of integral equations is approximated by (VIM) in [18] .Also in [11], (VIM) is applied for solving nonlinear system of ordinary differential equations. Thus, we can say Variational iteration method is a well known method to solve nonlinear equations. The convergence of (VIM) is discussed in [20]. In this work use (VIM) for solving nonlinear of mixed Volterra -Fredholm integral equations, for convenience we consider mixed Volterra - Fredholm integral equations as follows in [10] ,

$$
\begin{gathered}
u(x, y) \\
=f(x, y)+\int_{0}^{y} \int_{\Omega} G(x . y, s, t, u(s, t)) d s d t \\
(x, y) \in \Omega \times[0, T]
\end{gathered}
$$

where ,we suppose that $\Omega$ is colsed subset of $\mathrm{R}, \quad \mathrm{f}(\mathrm{x}, \mathrm{y})$ and $\mathrm{G}(\mathrm{x}, \mathrm{y}, \mathrm{s}, \mathrm{t}, \mathrm{u}(\mathrm{s}, \mathrm{t}))$ are analytic on

$\mathrm{D}=\mathbf{\Omega} \times[\mathbf{0}, \mathbf{T}]$,

$\widehat{D}$ respectively such that :

$$
\widehat{D}=\left\{(x, y, s, t) 0 \leq t \leq y \leq T(x, s) \in \Omega^{2}\right\} \times R
$$

\section{NONLINEAR OF MIXED VOLTERRA -FREDHOLM INTEGRAL}

The nonlinear intergral or differential equations are important in applied sciences and engineering. In [11 ] linear and nonlinear of ordinary equations are solved by using he is Variational iteration method and in [19 ] the exact solution of nonlinear of mixed Volterra -Fredholm integral equations , is approximated by Variational Iteration method .Now we consider this problem as follows :

$$
\begin{gathered}
u(x, y)=f(x, y)+\int_{0}^{y} \int_{\Omega} G(x, y, s, t, u(s, t)) d s d t \\
(x, y) \in \Omega \times[0, T]
\end{gathered}
$$


we try obtain an effective method for solving nonlinear of mixed Volterra -Fredholm integral equations ,

so we suppose $\Omega=[0,1]$

and by differentiating form both sides of Eq.(6) respect to y, we have

$$
\frac{\partial u}{\partial y}-\frac{\partial f}{\partial y}-\int_{0}^{1} G(x, y, s, y, u(s, y)) d s-\int_{0}^{y} \int_{0}^{1} \frac{\partial G}{\partial y} d s d t
$$$$
=\mathbf{0}
$$

now ,we use (VIM) for Eq.(7), so we obtain following

$$
\begin{aligned}
& u_{n+1}(x, y)=u_{n}(x, y)+\int_{0}^{y} \lambda\left[\frac{\partial u_{n}(x, \tau)}{\partial \tau}-\frac{\partial f(x, \tau)}{\partial \tau}\right. \\
&-\int_{0}^{1} G(x, \tau, s, \tau, u(s, \tau)) d s \\
&\left.-\int_{0}^{\tau} \int_{0}^{1} \frac{\partial G}{\partial \tau} d s d t\right] d \tau \\
& \mathbf{n}=\mathbf{0}, \mathbf{1}, \mathbf{2}, \ldots .
\end{aligned}
$$

by using the Variational theorem and effect $\delta$ in both sides of Eq.(10) and also with assume

$\delta u_{n+1}(x, y)=0$,

and

$$
\delta\left[-\int_{0}^{1} G(x, y, s, y, u(s, y)) d s-\int_{0}^{y} \int_{0}^{1} \frac{\partial G}{\partial y} d s d t\right]=0
$$

We have

$$
\begin{gathered}
u_{n+1}(x, y)=(1+ \\
=0
\end{gathered}
$$

By considering

$$
\delta u_{n+1}(x, y)=0
$$

optimal value of $\lambda$ is given by the following differential equation :

$$
\begin{aligned}
& 1+\lambda(y)=0 \\
& \left.\grave{\lambda}(\tau)\right|_{\tau=y}=0
\end{aligned}
$$

so, we get the Lagrange multiplier as follows :

$$
\lambda=-1
$$

by substituting $\lambda=-1$ in Eq.(8), we obtain an algebraic for finding solution of Eq.(5).

\section{NUMERICAL EXAMPLES}

In this section, we solve two examples of the nonlinear of mixed Volterra - Fredholm integral equations which have solved in [19 ] by using Collocation method numerical results show that our proposedmethod has a high accuracy and also VIM better than Collocation method.

\section{Example 1.}

Consider the following nonlinear of mixed Volterra -

Fredholm integral equations:

$$
u(x, y)=f(x, y)+\int_{0}^{y} \int_{0}^{1} u^{2}(t, s) d t d s
$$

where

$$
f(x, y)=-\frac{1}{5} y^{5}+\frac{1}{2} y^{4}-\frac{4}{9} y^{3}-y^{2}+2 x y
$$

with the exact solution

$$
u(x, y)=-y^{2}+2 x y
$$

for solvin the above equation, when $\lambda=-1$ we find the iteration sequences as follows:

$$
\begin{aligned}
& u_{n+1}(x, y)= \\
& u_{n}(x, y)-\int_{0}^{y}\left[\frac{\partial u_{n}(x, \tau)}{\partial \tau}-\frac{\partial\left(-\frac{1}{5} \tau^{2}+\frac{1}{2} \tau^{4}-\frac{4}{9} \tau^{3}-\tau^{2}+2 x \tau\right)}{\partial \tau}-\right. \\
& \left.\int_{0}^{1} u_{n}^{2}(t, \tau) d t-\int_{0}^{\tau} \int_{0}^{1} \frac{\partial}{\partial \tau}\left[u_{n}^{2}(t, s)\right] d t d s\right] d \tau \\
& \mathrm{n}=0,1,2, \ldots .
\end{aligned}
$$

by choosing $\mathrm{u} 0=0$ as an initial approximation .

$$
\begin{gathered}
u_{1}=\int_{0}^{y} \frac{\partial}{\partial \tau}\left(-\frac{1}{5} \tau^{5}+\frac{1}{2} \tau^{4}-\frac{4}{9} \tau^{3}-\tau^{2}+2 x \tau\right) d \tau \\
=-\frac{1}{5} y^{5}+\frac{1}{2} y^{4}-\frac{4}{9} y^{3}-y^{2}+2 x y \\
u_{2}=-\frac{1}{5} y^{5}+\frac{1}{2} y^{4}-\frac{4}{9} y^{3}-y^{2}+2 x y \\
-\int_{0}^{y}\left[\frac { \partial } { \partial \tau } \left(-\frac{1}{5} \tau^{5}+\frac{1}{2} \tau^{4}-\frac{4}{9} \tau^{3}-\tau^{2}\right.\right. \\
+2 x \tau) \\
-\frac{\partial}{\partial \tau}\left(-\frac{1}{5} \tau^{5}+\frac{1}{2} \tau^{4}-\frac{4}{9} \tau^{3}-\tau^{2}+2 x \tau\right) \\
-\int_{0}^{1}\left(-\frac{1}{5} \tau^{5}+\frac{1}{2} \tau^{4}-\frac{4}{9} \tau^{3}-\tau^{2}\right. \\
+2 t \tau)^{2} d t \\
-\int_{0}^{\tau} \int_{0}^{1} \frac{\partial}{\partial \tau}\left(-\frac{1}{5} s^{5}+\frac{1}{2} s^{4}-\frac{4}{9} s^{3}-s^{2}\right. \\
\left.+2 t s)^{2} d t d s\right] d \tau \\
u_{2}=-\frac{8}{45} y^{5}-y^{2}+2 x y+\frac{1}{275} y^{11}-\frac{1}{50} y^{10}+\frac{77}{1620} y^{9} \\
\\
\quad-\frac{1}{80} y^{8}-\frac{487}{2835} y^{7}+\frac{17}{54} y^{6}
\end{gathered}
$$

Numerical results fpr example 1 , is shown in Table 1 . for $\mathrm{N}=$ 4 and figures 1,2 and 3 .

Table 1: Numerical results of example 1.N = 4

\begin{tabular}{|l|l|l|l|}
\hline$(\mathrm{x}, \mathrm{t})$ & Exact sol & $\begin{array}{l}\text { Approximate } \\
\text { sol }\end{array}$ & Abs.error \\
\hline$(0,0)$ & 0 & 0 & 0 \\
\hline$(0.1,0.1)$ & 0.01 & 0.0099999 & $1 \times 10^{-11}$ \\
\hline$(0.2,0.2)$ & 0.04 & 0.03999999702 & $2.98 \times 10^{-9}$ \\
\hline$(0.3,0.3)$ & 0.09 & 0.08999992059 & $7.941 \times 10^{-8}$ \\
\hline$(0.4,0.4)$ & 0.16 & 0.1599992906 & $7.094 \times 10^{-7}$ \\
\hline$(0.5,0.5)$ & 0.25 & 0.2499965804 & $3.4196 \times 10^{-6}$ \\
\hline$(0.6,0.6)$ & 0.36 & 0.3599890815 & $1.09183 \times 10^{-}$ \\
\hline
\end{tabular}




\begin{tabular}{|l|l|l|l|}
\hline & & & 5 \\
\hline$(0.7,0.7)$ & 0.49 & 0.4899743999 & $\begin{array}{l}2.56001 \times 10^{-} \\
5\end{array}$ \\
& & & \\
\hline$(0.8,0.8)$ & 0.64 & 0.6399535465 & $\begin{array}{l}4.64535 \times 10^{-} \\
5\end{array}$ \\
& & & \\
\hline$(0.9,0.9)$ & 0.81 & 0.8099333359 & $\begin{array}{l}6.66641 \times 10^{-} \\
5\end{array}$ \\
& & & $\begin{array}{l}7.54765 \times 10^{-} \\
5\end{array}$ \\
\hline$(1,1)$ & 1.000 & 0.9999245235 & \\
\hline
\end{tabular}

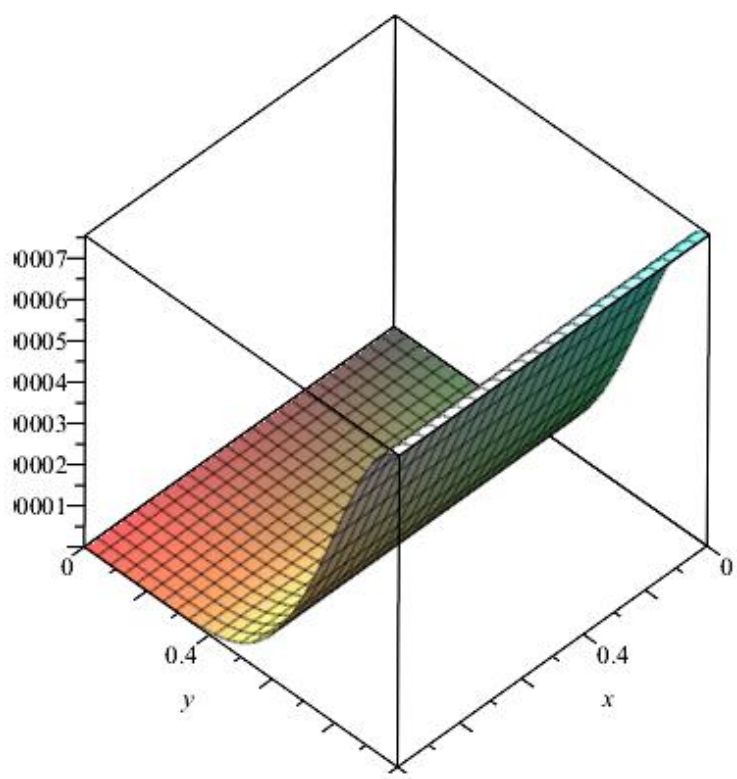

Figure 3: Absolute error of example 1

Example (2) Consider the following nonlinear of mixed Volterra-Fredholm integral equations:

$$
u(x, y)=f(x, y)+\left(x-y^{2}\right) \int_{0}^{y} \int_{0}^{1} u^{2}(t, s) d t d s
$$

Where

$$
f(x, y)=y^{2} \sin (x)-0.05453512866\left(x-y^{2}\right) y^{5}
$$

with the exact solution

$$
u(x, y)=y^{2} \sin x
$$

Figure 1: Approximate solution of example 1

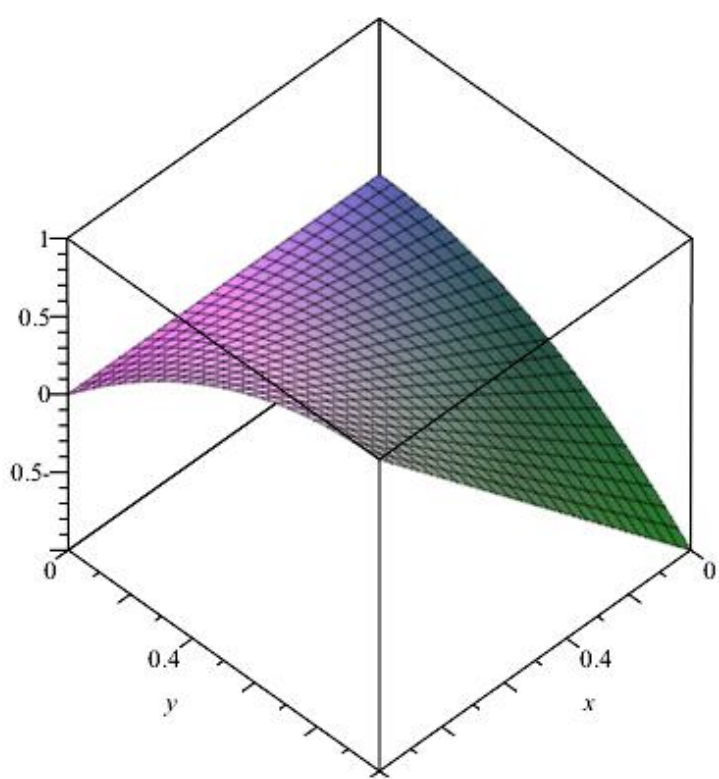

Figure 2:Exact solution of example 1 for solvin the above equation, when $\lambda=-1$ we find the iteration sequences as follows:

$$
\begin{aligned}
& =u_{n}(x, y) \\
& -\int_{0}^{y}\left[\frac{\partial u_{n}(x, \tau)}{\partial \tau}\right. \\
& -\frac{\partial\left(\tau^{2} \sin (x)-0.05453512866\left(x-\tau^{2}\right) \tau^{5}\right)}{\partial \tau} \\
& -\int_{0}^{1}\left(x-\tau^{2}\right) u_{n}^{2}(t, \tau) d t \\
& \left.-\int_{0}^{\tau} \int_{0}^{1} \frac{\partial}{\partial \tau}\left[\left(x-\tau^{2}\right) u_{n}^{2}(t, s)\right] d t d s\right] d \tau \\
& \mathrm{n}=0,1,2, \ldots \ldots
\end{aligned}
$$

by choosing $\mathrm{u} 0=0$ as an initial approximation

$$
\begin{aligned}
& u_{1}=\int_{0}^{y} \frac{\partial}{\partial \tau}\left(\tau^{2} \sin (x)-0.05453512866\left(x-\tau^{2}\right) \tau^{5}\right) d \tau \\
& =0.05453512866 y^{7}-0.05453512866 x y^{5}+y^{2} \sin (x)
\end{aligned}
$$

similarly u2.

Numerical results for example 2. is shown in Table 2. forN $=$ 2 and figures 4,5 and 6

Table 2: Numerical results of example 2, $\mathrm{N}=2$ 


\begin{tabular}{|c|c|c|c|}
\hline$(x, t)$ & Exact Sol & $\begin{array}{l}\text { Approximate } \\
\text { sol }\end{array}$ & Abs.error \\
\hline$(0,0)$ & 0 & 0 & 0 \\
\hline$(0.1,0.1)$ & 0.0009983341665 & 0.0009983341629 & $3.6 \times 10^{-12}$ \\
\hline$(0.2,0.2)$ & 0.007946771632 & 0.007946773232 & $1.6 \times 10^{-9}$ \\
\hline$(0.3,0.3)$ & 0.02659681860 & 0.02659676827 & $5.033 \times 10^{-8}$ \\
\hline$(0.4,0.4)$ & 0.06230693477 & 0.06230641571 & $5.1906 \times 10^{-7}$ \\
\hline$(0.5,0.5)$ & 0.1198563846 & 0.1198536043 & $2.7803 \times 10^{-6}$ \\
\hline$(0.6,0.6)$ & 0.2032712904 & 0.2032620438 & $9.2466 \times 10^{-6}$ \\
\hline$(0.7,0.7)$ & 0.31566666667 & 0.3156468072 & $1.98595 \times 10^{-5}$ \\
\hline$(0.8,0.8)$ & 0.4591078982 & 0.4590841580 & $2.37403 \times 10^{-5}$ \\
\hline$(0.9,0.9)$ & 0.6344947968 & 0.6344940476 & $7.492 \times 10^{-7}$ \\
\hline$(1,1)$ & 0.8414709848 & 0.8414709847 & $1 \times 10^{-10}$ \\
\hline
\end{tabular}

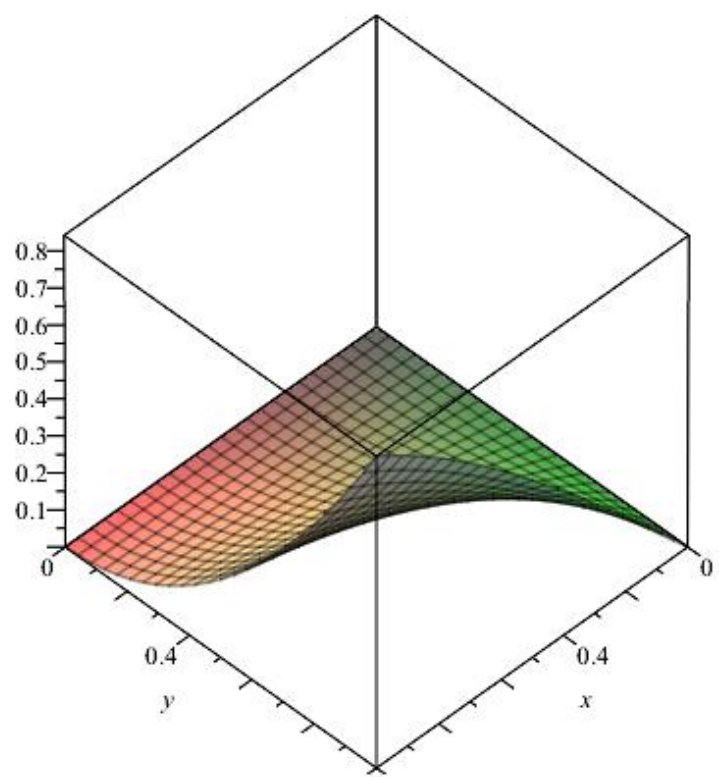

Figure 4: Approximate solution of example 2

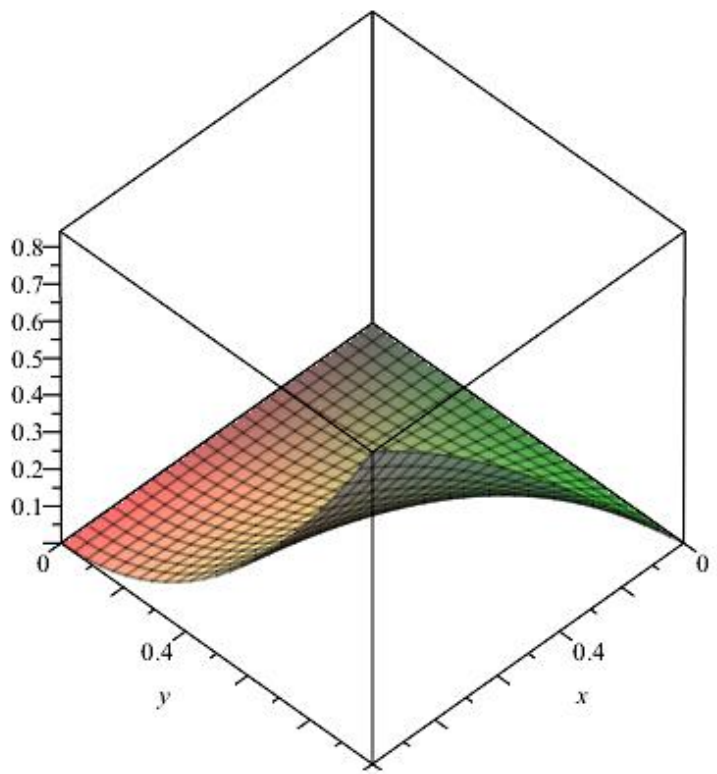

Figure 5: Exact solution of example 2 


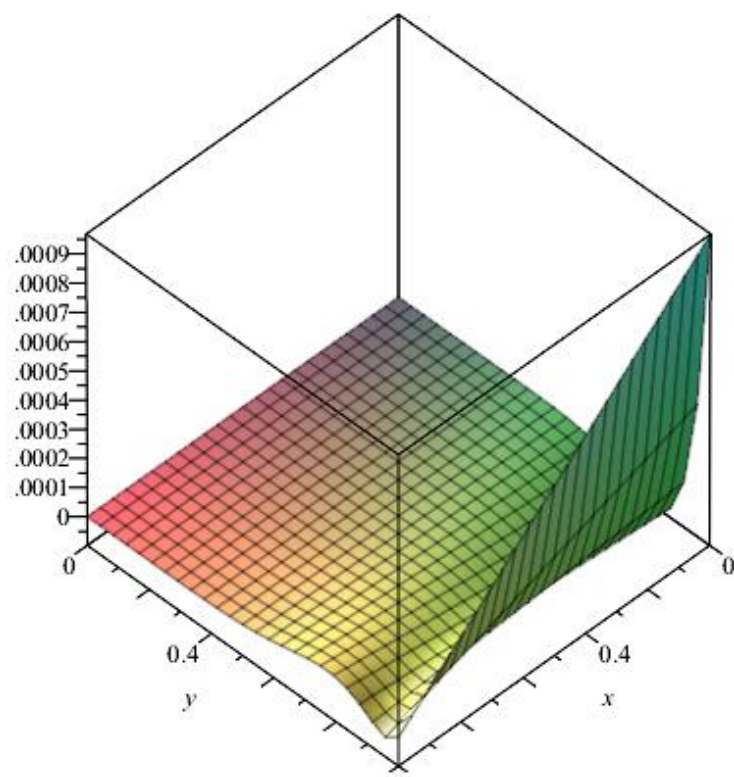

Figure 6: Absolute error of example 2

\section{CONCLUSION}

This paper concerns the numerical solutions of two dimensional volterra -Fredholm integral equations by using variational Iteration method, Examples 1 and 2 have been solve in [19] by collocation method, we show that our method is better than collocation method.

\section{REFERENCES}

[1] Darania . p and Ebadian . A ; Numerical solutions of the nonlinear two dimensional Volterraintegral equations .Newzealand Journal of mathematics .vol36(2007), 163 174.

[2] Nemati .S and Ordokhanyi .Y; Numerical solution of two dimensional nonlinear Volterraintegral equations by the Lagendre polynomials .J . SCI . Tarbiat Moalem University.vol11,No.2(2012), $195-210$.

[3] Saeedi . L, Tari . A and Masuleh . S . H . M ; Numerical solution of some nonlinearVolterra integral equations of the first kind.Applications and Applied Mathematics. vol8, (2013), $214-226$

[4] Bazm .S and Lima .P ; Numerical solutions of the nonlinear second kind two dimensionalVolterra integral equations using Extrapolation method .Numerical Analysis and Applied Mathematics, International conference(2010), 1195 - 1198

[5] Gholami .A ;Solving system of two dimensional nonlinear Volterra -Fredholm integro -differential equations by hes Variational Iteration method .Researcher (2015), $81-85$

[6] Erfanian .H .R and Mostahahsan .T ;Approximate solution of a class of nonlinear integralequations . The Journal of mathematics and computer science vol.3,No.3(2011), $278-286$
[7] Bahzadi .Sh .S ;The use of Iterative methods to solve two dimensional nonlinear Volterra-Fredholm integro differential equations .ISPACS , vol.(2012), 20pages

[8] Rabbani .M and Jamali .R ;solving nonlinear system of mixed Volterra -Fredholm integral equations by using Variational Iteration method . The Journal of mathematics and computerscience vol.5,No.4, (2012), $280-287$

[9] Borzabadi .A .H and Hedari .M ; A successive iterative approach for two dimensional nonlinearVolterra Fredholm integro -differential equations .Iranian Journal of Numerical Analysisand optimization .vol.4,No.1(2014), pp95 - 104

[10] Wazwaz . A ; Reliable Treatment for Mixed VolterraFredholm in Integral EquationsAppl.Math.comput.127(2002)405 - 414 .

[11] Biazar . J, Ghazvini . H ; He's variational iteration method for solving linearand nonlinear systems of ordinary differential equations, Applied mathematics andcomputation,191(2007)287-297.

[12] Finlayson . B . A , The method of weighted residuals and variational principles , Academicpress, (1972) Newyork

[13] He . J . H ; A new approach to nonlinear partial differential equations, Communications inNonlinear science and Numerical simulation,V ol.2,No.4(1997)230 -235 .

[14] He . J . H ; Nonlinear oscillation with fractional derivative and it's approximation, Int,conf.on vibration Engineering98,Dalian,China, (1998).

[15] He . J . H, Variational iteration method for nonlinear and it's applications, Mechanics andpractice,20, (1)(1998)30 - 32(inchinese)

[16] He . J . H ; Variational Iteration method - a kind of nonlinear analytical technique:Someexamples, Int.Journal of Nonlinear Mechanics,34(1999)699 - 708.

[17] Inokuti . M ; General use of the Lagrange multiplier in in nonlinear mathematicalphysics,in: S. Nemat-nasser(Ed.), Variational Method in Mechanics of solids, Progamonpress, oxford, (1978)156- 16210

[18] Xu. Lan ; Variational iteration method for solving integral equations, computers and Mathematicswith Applications,54(2007)1071 - 1078

[19] Abdollah Borhanifar. and Khadijeh Sadri, Numerical solution for systems of two dimensionalintegral equations by using Jacobi operational collocation method.Sohag J. Math. 1, No. 1,15-26 (2014).

[20] Tatari . M , Dehghan . M ; On the Convergence of He's Variational Iteration Method, J.comput.Appl.Math,207(2007)121-128 